
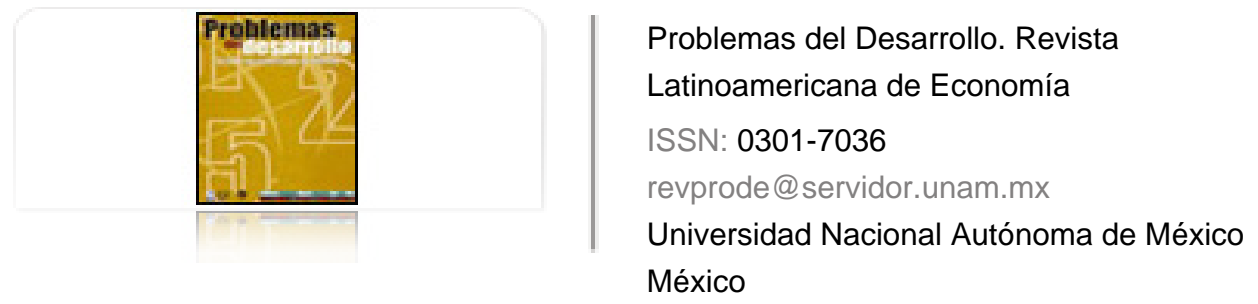

Piña Zambrano, Henri Jesús; Morales Espinoza, Agustín

ALOE EN VENEZUELA: DE LA CADENA DE VALOR AL DISTRITO INDUSTRIAL

Problemas del Desarrollo. Revista Latinoamericana de Economía, vol. 41, núm. 160, enero-marzo, 2010, pp. 187-208

Universidad Nacional Autónoma de México

Distrito Federal, México

Disponible en: http://www.redalyc.org/articulo.oa?id=11820132009

Cómo citar el artículo

- Número completo

- Más información del artículo

Página de la revista en redalyc.org

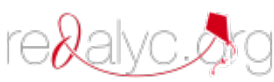

Sistema de Información Científica

Red de Revistas Científicas de América Latina, el Caribe, España y Portugal Proyecto académico sin fines de lucro, desarrollado bajo la iniciativa de acceso abierto 


\title{
ALOE EN VENEZUELA: DE LA CADENA DE VALOR AL DISTRITO INDUSTRIAL
}

\author{
Henri Jesús Piña Zambrano* \\ Agustín Morales Espinoza**
}

Fecha de recepción: 8 de junio de 2009. Fecha de aceptación: 10 de octubre de 2009.

\section{Resumen}

Desde la perspectiva del estudio de los conglomerados, se contrastaron las aportaciones teóricas acerca de los conglomerados y el funcionamiento y desempeño de la cadena del aloe en el Estado Falcón (Venezuela), partiendo de la hipótesis de que esta cadena ha seguido un patrón evolutivo sustentado en el legado histórico de recursos que han favorecido la siembra y explotación del aloe en el estado. Sin embargo, se considera un escaso aprovechamiento de este patrimonio como estrategia para conformar un eficiente y competitivo conglomerado agroindustrial. La investigación se sustenta en estudios realizados entre 2003 y 2007 en las zonas productoras del aloe. Se identifican en la cadena los elementos típicos de un conglomerado (proximidad geográfica, redes, innovación e institucionalidad) bajo un patrón organizativo que no promueve la consolidación del conglomerado. En la cadena del aloe no termina de crearse la atmósfera industrial necesaria para iniciar la districtualización de esta actividad productiva.

Palabras clave: conglomerado, aloe, Falcón, distrito industrial.

* Coordinador de la Unidad Estratégica de Análisis Agroalimentario, Universidad Francisco de Miranda, Estado Falcón, Venezuela. Correo electrónico: henripina@ gmail.com

** Coordinador de la Unidad de Investigaciones Agroalimentarias de la Facultad de Agronomía, Instituto de Economía Agrícola y Ciencias Sociales, Universidad Central de Venezuela. Correo electrónico: moralesa@ agr.ucv.ve 


\section{Abstract}

From a perspective of studying conglomerates, the theoretical contributions on conglomerates and the functioning and performance of the aloe chain in the state of Falcón (Venezuela) are contrasted, starting by using the hypothesis that this chain has followed an evolutionary pattern sustained by the historical legacy of resources that has favored the planting and exploitation of aloe in this state. However, the exploitation of this resource is thought to be ineffective as a strategy to form an efficient and competitive agro-industrial conglomerate. The study is based on work carried out between 2003 and 2007 in the aloe producer zones. Identified in the chain are the elements typical of a conglomerate (geographical proximity, networks, innovation and institutions) in an organizational pattern that does not promote the consolidation of the conglomerate. In the aloe chain the industrial atmosphere needed to begin the districting of this productive activity is still being built.

Key words: conglomerate, aloe, Falcón, industrial district.

\section{Résumé}

Depuis la perspective de l'étude des conglomérats, les apports théoriques sur ces derniers sont mis en contraste avec le fonctionnement et les résultats de la chaîne de l'aloès dans l'État de Falcón au Venezuela, en partant de l'hypothèse que cette chaîne a suivi un schéma évolutif fondé sur un legs historique de ressources qui ont favorisé la plantation et l'exploitation de l'aloès dans le dit état. Néanmoins, il est considéré que ce patrimoine est peu mis à profit dans le cadre d'une stratégie pour constituer un conglomérat industriel efficace et compétitif. Cette recherche se base sur des études réalisées entre 2003 et 2007 dans les zones productrices d'aloès. On peut identifier la chaîne des éléments typiques d'un conglomérat (proximité géographique, réseaux, innovation et caractère institutionnel) dans un schéma d'organisation qui ne promeut pas sa consolidation. Dans la chaîne de l'aloès, il n'a pas encore été créé le contexte industriel nécessaire pour que voit le jour un district industriel sur cette activité productive. Mots clés: conglomérat, aloès, Falcón, district industriel.

\section{Resumo}

Da perspectiva do estudo dos conglomerados, contrastaram-se as contribuições teóricas sobre os conglomerados e o funcionamento e desempenho da cadeia da babosa no Estado Falcón (Venezuela), partindo da hipótese de que esta cadeia tem seguido um padrão evolutivo sustentado no legado histórico de recursos que favorecem o plantio e a exploração da babosa no estado. No entanto, considera-se um escasso aproveitamento deste patrimônio como estratégia para conformar um eficiente e competitivo conglomerado agroindustrial. A pesquisa se apóia em estudos realizados entre 2003 e 2007 nas zonas produtoras de babosa. Identificam na cadeia os elementos típicos de um conglomerado (proximidade geográfica, redes, inovação e institucionalidade) sob um padrão organizativo que não promove a consolidação do conglomerado. $\mathrm{Na}$ cadeia da babosa não se termina de criar a atmosfera industrial necessária para iniciar a distritalização desta atividade produtiva.

Palavras-chave: conglomerado, babosa, Falcón, distrito industrial.

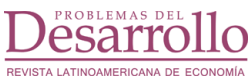

Vol. 41, núm. 160, enero-marzo / 2010 


\section{Introducción}

os procesos globales de desarrollo conllevan la posibilidad de ubicar en cual-
quier lugar del planeta a proveedores especializados para soportar procesos
productivos innovadores, incluso en las regiones más apartadas del mundo. La consecuencia principal de esta acción es la pérdida de capacidad competitiva de los agentes locales que carecen de las destrezas requeridas por las empresas demandantes de sus insumos. Así, aunque no todos los territorios son agronómicamente aptos para el cultivo del aloe, el procesamiento y elaboración de bienes finales de consumo pueden localizarse en cualquier lugar del mundo, siempre que lo permitan los costos operativos.

Adicionalmente, es importante aclarar que siempre existirán territorios con condiciones económicas, sociales o políticas más apropiadas para la producción del aloe que Falcón, por lo cual se convierten en potenciales áreas de inversión. Estas zonas compiten directamente con una actividad arraigada en la historia del estado, pero que ante la dinámica mundial ha exhibido una pobre capacidad de respuesta orientada a un mayor nivel de competitividad y hoy en día está amenazada por el surgimiento de nuevos enclaves de producción. Claros ejemplos de ello son las plantaciones de aloe localizadas en México, Costa Rica o República Dominicana.

El propósito del presente artículo es aplicar, como instrumento metodológico, el esquema teórico de los conglomerados ${ }^{1}$ para analizar la cadena productiva del aloe en Falcón (Venezuela), y derivar del mismo potenciales elementos clave que es necesario atender para evolucionar de una actividad tradicional y semiindustrial, hacia un competitivo conglomerado industrial sostenido en los atributos de este sector productivo.

Para tal fin, se revisan las principales aportaciones del estudio de los conglomerados, con el objetivo de organizar un patrón evolutivo de estas estructuras industriales, resaltando los actores participantes, conexiones y grado de influencia en un

1 En atención a la vasta profusión de términos sinónimos y complementarios relacionados con los conglomerados de empresas (cluster, aglomeraciones, tramas o distritos industriales, entre otros), de acuerdo con la naturaleza del estudio conducido, este artículo adoptará el término conglomerado, salvo cuando se indique lo contrario.

Como término genérico, conglomerado permite designar las distintas variantes de este esquema de organización productiva, indicando en su momento, la precisión teórica del mismo término según sus atributos distintivos. Por tanto, se reconoce la diferenciación entre sus distintas acepciones y particularidades; sin embargo, su uso a lo largo del artículo se hace en forma genérica.

Vol. 41, núm. 160, enero-marzo / 2010

Desarrorollo 
marco espacio-temporal, donde el territorio se presenta como un escenario donde se materializan todas y cada una de las acciones de los agentes del aloe. Por tanto, el territorio se concibe como un complejo entramado de relaciones (vinculaciones) entendidas como regímenes (patrones) en la vida cotidiana, prácticas sociales colectivas donde los individuos se involucran (tácitamente) en una red (horizontal, vertical y transversal) de interacción social, cimentadas a partir de expectativas sociales mutuas establecidas por ellos mismos (Piña, 2008; Sotolongo, 2007).

\section{Cadena productiva del aloe: un caso de estudio}

El Estado Falcón se ubica geográficamente al norte de América del Sur, frente al Mar Caribe. Posee una extensión de $24800 \mathrm{~km}^{2}$ y una población superior a los 700000 habitantes. Su clima es variado, pasando del xerófilo en la llanura costera $\left(28^{\circ}\right.$ a $\left.35^{\circ} \mathrm{C}\right)$, al de montaña en la sierra $\left(23^{\circ}\right.$ a $\left.14^{\circ} \mathrm{C}\right)$. En 10 de sus 25 municipios, principalmente en los de mayor carácter semiárido, se cultiva el aloe.

En Falcón, este cultivo se remonta a la llegada de los españoles a América; los habitantes de la época poseían un vasto conocimiento de las propiedades medicinales de la planta. El aloe fue introducido al estado desde Curaçao, a donde llegó desde Barbados producto del comercio marítimo que mantenían los pobladores de estas localidades. Las primeras plantaciones comerciales de importancia datan de 1870, pero no fue hasta 1920 cuando comenzó a cultivarse en mayor escala (González, 1999).

Desde entonces, el aloe se explota de manera artesanal en plantaciones de pequeña escala; luego se somete a un procesamiento agroindustrial rudimentario para extraer el principal bien comercializable: la pasta acíbar (exudado de la hoja), deshidratada mediante cocción hasta alcanzar un sólido de color negruzco con $4 \%$ de humedad. Se comercializa en empaques de madera de $60 \mathrm{~kg}$ de peso cada uno (Piña, 2006; Piña, Lugo, Azócar y Romero, 2005).

Este subproducto tuvo un importante auge entre las décadas de 1970 y 1980 , cuando se llegó a exportar hasta 13\% del mercado mundial, por entonces estimado en cerca de US \$2 millones. Sin embargo, en la actualidad Falcón ha sido desplazado por importantes plantaciones tecnificadas establecidas en países del Caribe, en donde se utilizan modernas prácticas de cultivo y las plantaciones se han orientado hacia la obtención de gel (cuerpo mucilaginoso resultante de la eliminación de la epidermis de la hoja, filtrado y estabilizado) en diferentes presentaciones (desde gel $1 \mathrm{X}$ hasta gel liofilizado $)^{2}$, vistas sus amplias aplicaciones en las industrias cosméticas, médicas,

2 En la gama del gel, el gel 1X corresponde al producto básico; el liofilizado, al premium.

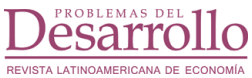

Vol. 41, núm. 160, enero-marzo / 2010 
agroalimentarias y farmacéuticas. Este mercado ha evolucionado significativamente durante los últimos años y mantiene una proyección de crecimiento no menor a $12 \%$ interanual, estimándose un mercado global de US \$65 millones en productos primarios (hojas, gel e hijuelos) y más de US \$200 millardos en bienes finales (champú, lociones, bebidas y medicamentos) (Piña, 2005).

En contraste, aunque en los últimos 10 años la superficie de explotación del aloe en Falcón ha aumentado hasta llegar a superar las 4600 hectáreas en la actualidad, en su mayoría éstas son explotadas siguiendo los procedimientos ancestrales, establecidos desde la época en que el cultivo llegó a estas tierras, desaprovechando la construcción de un conglomerado agroindustrial en las diferentes zonas de explotación, que contribuyera al desarrollo rural y permitiera elevar el nivel de vida de la población de las zonas del estado donde se cultiva el rubro, regiones caracterizadas por sus condiciones de marginalidad económica, que en algunos casos alcanzan niveles de desempleo cercanos a 70\%, y cuya principal actividad se sustenta en la cría extensiva de caprinos, actividades comerciales menores y la eventual explotación de hortalizas de secano (Piña, 2004).

En la cadena del aloe participan, fundamentalmente, ocho grupos de actores (véase cuadro 1); tres de ellos, los productores, la agroindustria y los comercializadores, desempeñan una función determinante en la dinámica productiva. En los últimos años, los productores se han dado a la tarea de rescatar antiguas asociaciones y crear nuevas, con la finalidad de que sirvan de interlocutores válidos ante el resto de los actores. Las gestiones de los productores ante los organismos oficiales han reportado el otorgamiento de programas de financiamiento por cerca de US \$4 millones en los últimos seis años, que principalmente se han destinado a la siembra de nuevas plantaciones y a la ampliación de las existentes.

En cuanto al grupo de agroindustrias, sólo hay dos establecidas en el estado, ambas con importante participación de capital extranjero que les ha facilitado el acceso a tecnología, capital y mercados. La más reciente de estas agroindustrias ha creado un novedoso e interesante programa de apertura de compra hacia los productores, que ha comenzado a dibujar un nuevo escenario al llevar a cabo acciones transparentes de compra-venta con proveedores locales de hojas para gel, rompiendo así el tradicional sesgo mantenido por la otra empresa.

Sin embargo, aún es muy escasa la coordinación vertical en el ámbito agroindustrial en la cadena. Este eslabón ha enfrentado serios problemas de suministro de materia prima en términos de regularidad, calidad y volumen, al punto de que en el pasado se vio obligado a importar hojas para mantener tanto sus niveles de producción como el desarrollo de sus propias plantaciones. 
Cuadro 1

Agentes económicos y actividades desempeñadas

\begin{tabular}{|c|c|c|c|}
\hline Agente & Actividad desempeñada & Perfil del agente & Grupo estratégico \\
\hline $\begin{array}{c}\text { Proveedores de insumos } \\
\text { y servicios }\end{array}$ & $\begin{array}{l}\text { Preparación de tierras } \\
\text { Venta de insumos } \\
\text { Venta de hijos (semillas) }\end{array}$ & $\begin{array}{l}\text { Pequeñas dimensiones } \\
\text { Bajo impacto económico } \\
\text { Actividad eventual }\end{array}$ & $\begin{array}{l}\text { Productores } \\
\text { Empresa servicios } \\
\text { Casas comerciales } \\
\text { Ejecutivo }\end{array}$ \\
\hline Productores & $\begin{array}{l}\text { Siembra y mantenimiento } \\
\text { Cosecha } \\
\text { Mercadeo de la producción }\end{array}$ & $\begin{array}{l}\text { Conocimiento del cultivo } \\
\text { Desconocimiento del mercado } \\
\text { Alta motivación al cambio }\end{array}$ & $\begin{array}{l}12 \text { asociaciones } \\
950 \text { socios } \\
5600 \text { has }\end{array}$ \\
\hline Procesadores & $\begin{array}{l}\text { Cocción del acibar } \\
\text { Elaboración de la pasta }\end{array}$ & $\begin{array}{l}\text { Alta especialización } \\
\text { Número reducido }\end{array}$ & Pequeña escala \\
\hline Manufactura artesanal & $\begin{array}{l}\text { Compra de bienes primarios } \\
\text { Procesamiento artesanal } \\
\text { Venta de bienes finales }\end{array}$ & $\begin{array}{l}\text { Bienes diferenciados } \\
\text { Nichos de mercado cautivos } \\
\text { Demanda en ascenso }\end{array}$ & $\begin{array}{l}\text { Pequeña escala } \\
\text { Número reducido }\end{array}$ \\
\hline Agroindustria local & $\begin{array}{l}\text { Compra de acibar y hojas } \\
\text { Concentración de acibar } \\
\text { Exportación de bienes }\end{array}$ & $\begin{array}{l}\text { Dominio de la tecnología } \\
\text { Conocimiento del mercado } \\
\text { Poca integración vertical }\end{array}$ & $\begin{array}{l}\text { PIZCA } \\
\text { NATURAVEN }\end{array}$ \\
\hline Comerciantes & $\begin{array}{l}\text { Acopio local de producción } \\
\text { Venta a brokers } \\
\text { Almacenamiento }\end{array}$ & $\begin{array}{l}\text { Conocimiento de mercado } \\
\text { Monopsonio imperfecto } \\
\text { Tensa relación productores }\end{array}$ & 3 agentes \\
\hline Organismos oficiales & $\begin{array}{l}\text { Marco jurídico } \\
\text { Financiamiento } \\
\text { Organización } \\
\text { Capacitación } \\
\end{array}$ & $\begin{array}{l}\text { Función activa } \\
\text { Circuito estratégico } \\
\text { Importante apoyo }\end{array}$ & $\begin{array}{l}\text { Universidad } \\
\text { Ejecutivo } \\
\text { Centros I \& D } \\
\text { Grupo de trabajo }\end{array}$ \\
\hline Industria manufacturera & $\begin{array}{l}\text { Compra de bienes intermedios } \\
\text { Elaboración de bienes finales }\end{array}$ & $\begin{array}{l}\text { Altos estándares de calidad } \\
\text { Fuerte relación proveedores }\end{array}$ & $\begin{array}{l}\text { Cosmética } \\
\text { Médica } \\
\text { Farmacéutica } \\
\text { Alimentaria } \\
\end{array}$ \\
\hline Consumidor final & Adquisición y uso de bienes finales & $\begin{array}{l}\text { Atractivo mercado } \\
\text { Alta tendencia naturista }\end{array}$ & $\begin{array}{l}\text { Nicho de mercado } \\
\text { Bien diferenciado }\end{array}$ \\
\hline
\end{tabular}

Fuente: Piña, 2006.

Esta situación tiene su origen precisamente en el esquema tradicional de producción primaria del aloe, orientado a la extracción de acíbar debido a las condiciones semiáridas del lugar donde se cultiva y, por tanto, a la escasa disponibilidad de agua para riego que permitiría obtener hojas para gel. En definitiva, hay una gran incompatibilidad entre esquemas de producción primaria y procesamiento agroindustrial. En cualquier caso, la agroindustria del aloe no cuenta con la capacidad (de ser la situación) de procesar la totalidad de la producción de hojas de aloe en Falcón, y el problema radica en la calidad de la materia prima utilizada.

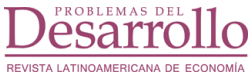


Por otro lado, se espera la puesta en marcha de siete plantas procesadoras de capital público, cinco de ellas se orientarán a la concentración, una al procesamiento de gel y otra a la refinación del acíbar. Estas plantas estarán distribuidas estratégicamente en la geografía de los municipios productores de aloe.

La actuación de los comercializadores de pasta da origen a una situación muy particular de oligopsonio imperfecto: sólo hay tres compradores y ellos son quienes imponen las reglas del juego. Estos agentes mantienen fuertes lazos con sus compradores en el extranjero y encauzan el comportamiento del mercado hacia sus intereses particulares. Por otro lado, existen compradores principalmente enfocados en el mercado nacional, que manejan pequeños volúmenes de producto y no llegan a ejercer un peso específico importante en la cadena.

Los actores oficiales se han dedicado fundamentalmente a la capacitación de los productores en temas de manejo agronómico y a la promoción de nuevas estructuras organizativas. Asimismo, han adelantado programas de financiamiento y son responsables de la construcción de siete plantas procesadoras, con una inversión de US \$9.5 millones. Los centros de I\&D y las universidades han participado de manera activa y protagónica, pero sus resultados no terminan de responder a las exigencias de una actividad productiva de la magnitud que representa el aloe en el mundo actual. No hay coordinación de actores ni la promoción de una meta en común.

En suma, la cadena del aloe es una actividad de tipo marginal en contraste con su atractivo mercado, y desaprovecha las potencialidades de cada uno de sus componentes. Mientras se pueda configurar una estrategia coordinada y articulada en función de un argumento vertebrador con el cual se identifiquen sus actores, se podría iniciar un importante y significativo esquema evolutivo hacia un conglomerado agroindustrial, pasando de la producción de commodities hacia bienes finales de alto valor agregado.

\section{Conglomerados: visión teórica}

Los conglomerados de empresas han sido un importante centro de atención y estudio por parte de profesionales de distintas especialidades, resultando en un prolífico aporte al conocimiento, desempeño y evolución de estas estructuras industriales. Conglomera$d o$ es un concepto que ha sido objeto de multitud de definiciones y enfoques de acuerdo con la escuela y el contexto particular en el cual se ha desarrollado cada noción. Sin embargo, dentro de esta amplia variedad conceptual destacan varios elementos en común (véase figura 1): proximidad geográfica, redes, innovación e instituciones.

Las economías externas se usaron al inicio de los estudios sobre conglomerados para explicar el agrupamiento de empresas (principalmente, Pymes) en un área geográ- 
Figura 1

Visión teórica del conglomerado

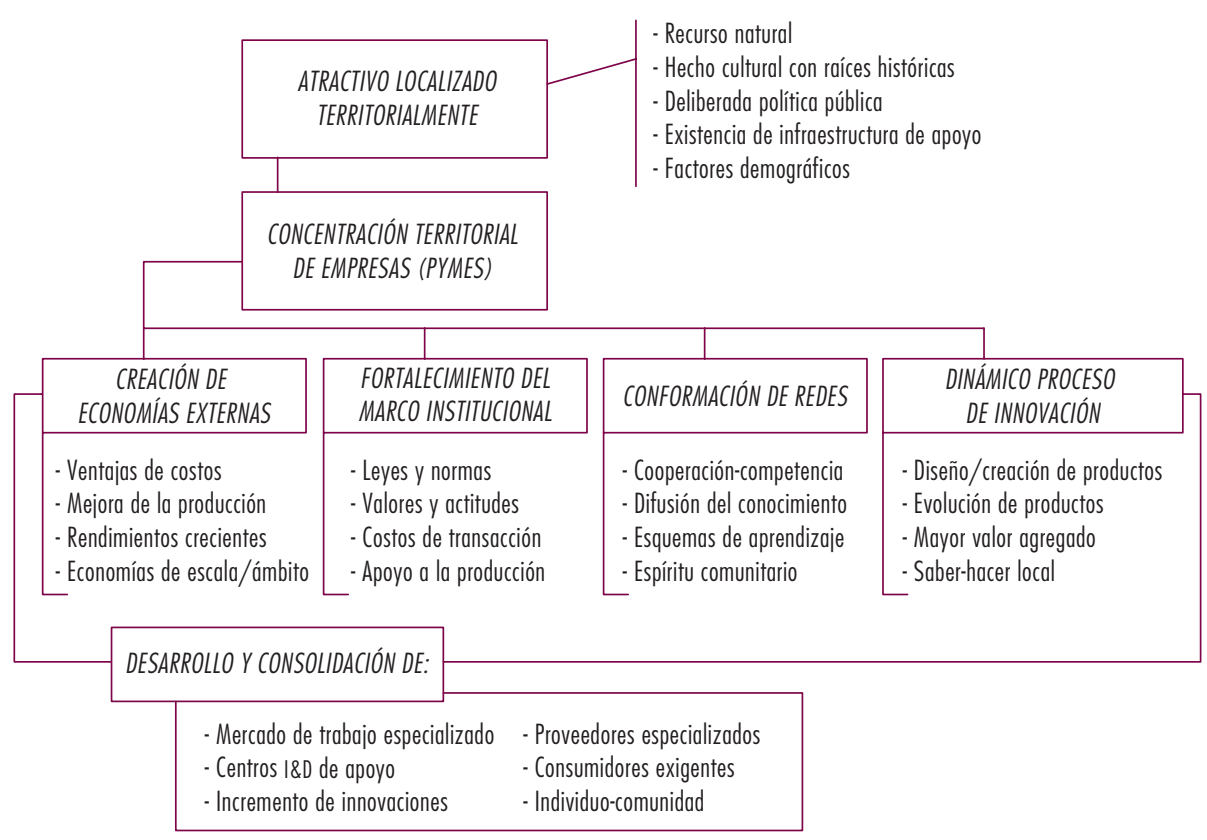

fica en particular. Estas economías se generan a partir de la cercanía de distintos agentes interactuando en procesos propios de su actividad económica, situación que se resumió en el término atmósfera industrial, empleado para describir el entorno socioeconómico de donde emergen beneficios provenientes de procesos de imitación, aprendizaje, innovación y adopción tecnológica. Muchos de estos procesos se materializan a partir de la cooperación entre agentes del distrito industrial, tal como se denominó al conglomerado de empresas (Marshall, 1920).

En este mismo contexto, en un sentido amplio, se identificaron economías de aglomeración, como beneficios obtenidos por una empresa al participar en economías locales, donde el tamaño de la aglomeración (consumidores y proveedores) le permite generar bienes diferenciados, mediante economías internas de escala, derivados de la posibilidad de la empresa de seleccionar de entre el conglomerado a proveedores especializados a bajo costo, así como de beneficiarse de la difusión del conocimiento generado en éste. Así, el conglomerado de empresas en un espacio determinado genera economías externas localizadas, dirigidas a reducir costos de producción.

Por su parte, las economías de escala están referidas a la capacidad de la empresa de reducir sus costos medios de producción o aumentar sus beneficios, lo que obedece

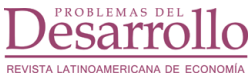


a la disminución del costo de insumos utilizados en el proceso productivo (al adquirir mayores volúmenes de los mismos), a la incorporación de mejoras tecnológicas o la especialización del capital humano. De ahí la importancia de los conglomerados como proveedores de beneficios a las empresas participantes (mano de obra especializada, innovación tecnológica y/ o infraestructura de apoyo), resaltando la proximidad geográfica entre empresas para crear condiciones económicas propicias para su consolidación (atmósfera industrial).

El hallazgo marshalliano (atmósfera industrial) evidenció un continuo y rápido flujo de actividades de innovación de un agente a otro, en donde se reconocen y valoran las buenas ideas entre agentes. En este esquema de organización industrial, un incremento en la producción de bienes genera tanto economías internas como externas de escala e implica la oferta de trabajadores y proveedores especializados, así como clientes y consumidores informados: un mercado de trabajo especializado.

Otro tratamiento empírico sobre los conglomerados de empresas corresponde a los distritos industriales italianos, los cuales destacan un simple agrupamiento de empresas donde además del agrupamiento (Pymes, preferentemente) hay una marcada presencia de valores y actitudes de las personas haciendo vida en el distrito. En este enfoque teórico, los individuos y las empresas constituyen un modelo comunitario de trabajo, atendiendo a la especificidad económica de cada uno. Bajo la concepción italiana, la atención se centra en la integración del aparato productivo local con la comunidad, con énfasis en el origen del conocimiento, los valores, las instituciones y las formas de comportamiento, modeladoras de una personalidad sociocultural del distrito; así, el objeto de estudio se centra en el cambio de congruencia en tiempo y espacio entre los procesos elementales en los cuales un distrito puede descomponerse (Becattini, 2002).

De la concepción italiana del distrito industrial, surge el término districtualización para referirse no a un evento puntual sino a la constante modulación en tiempo y espacio de un conjunto de procesos. Así se puede adoptar una perspectiva de la industrialización orientada hacia la historia concreta de una localidad y no tanto hacia el desarrollo de un sector industrial. Los sistemas locales en los cuales los aspectos económicos, productivos y socioculturales se combinan de tal manera que aseguran su reproducción, son parte de un todo social, cada cual como un todo más pequeño en sí mismo (Becattini, 2002).

Un tercer enfoque de los conglomerados de empresas se refiere a los clúster, que hace hincapié en la integración de unidades productivas. Desde esta perspectiva teórica, los conglomerados corresponden a un determinado conjunto de empresas (industrias) e instituciones conexas afines a una actividad económica, interrelacio- 
nadas en función de rasgos comunes y complementarios entre sí, sobre un espacio geográfico particular, ya sea local, regional o nacional (Porter, 1998).

Como se aprecia, el estudio de los conglomerados evolucionó conforme se incorporaron distintas disciplinas a su análisis. De manera específica, un punto de partida corresponde al tratamiento de las economías externas (teoría clásica y neoclásica), centrado en las economías agrarias e industriales basadas en recursos naturales como factor de localización industrial, hasta la teoría institucional (North, 2001) y el aporte de la nueva geografía económica (Krugman, 1979).

Complementariamente, otros estudios destacan el carácter dinámico y complejo de esta actividad económica, centrándose en la creciente importancia de la economía del conocimiento y la complejidad de relaciones de agentes y actividades, dirigiendo un proceso de cambio tecnológico. Se resalta como nuevo factor competitivo la capacidad de pensar, articular y generar ese conocimiento, junto a la función del territorio en una economía global, estimulando el papel del tejido productivo local sobre el territorio, donde y con el cual actúa ese tejido productivo (Boscherini y Poma, 2000).

A partir de los estudios realizados a lo largo de la historia de los conglomerados, se identifican elementos distintivos de estas estructuras, como la concentración (proximidad) geográfica, múltiples actores, actividades especializadas, entorno institucional, innovación, trabajo especializado, cooperación y competencia. Cada cualidad no necesariamente está presente en un conglomerado, dada la condición de singularidad de cada proceso evolutivo. Cada conglomerado es único y diferente a los otros, incluso entre los de una misma actividad económica. Sin embargo, éstos han sido elementos clave para el éxito de un determinado conglomerado en un punto particular de la historia y cada uno de ellos ha sido importante para su éxito, según el tipo de conexión con el resto de los factores y las condiciones particulares del momento. En definitiva, no hay un orden de ocurrencia, todo depende de la singular identidad de cada conglomerado.

\section{Metodología y datos}

Al contrastar el diseño teórico del conglomerado y el análisis de caso del aloe, se hipotetiza la presencia en diversas localidades de Falcón (territorio) de un patrón evolutivo sustentado parcialmente en un legado histórico (patrimonio natural propio) de recursos (sociales, económicos y culturales) que han favorecido la siembra y explotación del aloe en el estado. Sin embargo, se considera un escaso aprovechamiento de este patrimonio, que desperdicia una significativa oportunidad para establecer un conglomerado agroindustrial en torno a estas plantaciones. Así, la sistematización productiva de zonas de explotación de aloe en Falcón puede llegar a generar procesos 
de crecimiento y desarrollo al articular organizada y coherentemente a sus actores (redes), con la finalidad de crear un ambiente proclive (externalidades) para generar y establecer innovaciones tecnológicas (nuevo conocimiento) a partir del saber-hacer local acumulado en el transcurso de la evolución histórica del cultivo en el estado.

Partiendo de los supuestos de estudio expuestos y de la ausencia de un cuerpo teórico-epistemológico para analizar e interpretar el comportamiento del conglomerado aloe, se formularon las siguientes hipótesis de trabajo:

1. En las localidades productoras de aloe en Falcón existen condiciones ideales para crear un conglomerado agroindustrial, lo que amerita identificarlas, precisarlas, cuantificarlas y cualificarlas para estimar la pertinencia y sustentabilidad del conglomerado aloe en el mediano y largo plazos.

2. La ausencia de un conglomerado aloe en Falcón se debe principalmente a la escasa identidad y cohesión de los agentes del cultivo (no existe un argumento vertebrador), a la falta de visión compartida e internalizada por todos por igual. No hay formalidad en la adecuada interacción social para generar efectivas y eficientes redes productivas promotoras de mecanismos de articulación e intercambio entre agentes en pro de la creación de economías a escala, dirigidas a fomentar el crecimiento y desarrollo del conglomerado.

3. El establecimiento del conglomerado aloe, entendido como la compleja articulación de actores socioeconómicos, debe constituir un factor clave dentro de una estrategia de desarrollo territorial rural, al actuar como mecanismo activador de recursos latentes localizados territorialmente, en vista de los beneficios generados a partir de su dinámica productiva, en los ámbitos local e internacional.

4. A pesar de la noción nominal de la importancia del cultivo del aloe para las áreas rurales del estado, su relevancia real no ha sido suficientemente sólida ni determinante para justificar la sistematización de un entorno institucional promotor del ambiente requerido para viabilizar el conglomerado en Falcón.

Para dar respuesta a estos planteamientos, se parte del conjunto de investigaciones realizadas entre 2003 y 2007 (Piña, 2006; 2005; Piña et al., 2005; Piña, 2004), en las cuales se aplicaron cuestionarios en la modalidad de entrevistas estructuradas a los agentes clave de la cadena, incluyendo herramientas tipo Delphi. Asimismo, se recurrió a la consulta de especialistas afines a las variables de estudio, junto al análisis de caso como herramienta para proveer el andamiaje empírico del objeto de investigación.

Vol. 41, núm. 160, enero-marzo / 2010

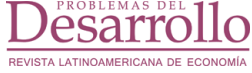




\section{De la cadena al conglomerado}

\section{a. Concentración geográfica y externalidades}

El impacto de la concentración geográfica y las externalidades que de ella se derivan ha sido explicado por las teorías clásica y neoclásica, enfatizando en el uso localizado de recursos naturales como factores de producción tradicionales. En un principio, la noción de territorio respondió a un concepto meramente teórico, y la localización de empresas se explicó mediante modelos estáticos y supuestos simplificados acerca de la competitividad de los mercados y los retornos constantes a escala. Más recientemente, por medio de la nueva geografía económica (Krugman, 1979), se ha tratado de explicar la localización industrial, sin embargo, sólo se ha focalizado en la tendencia de las empresas a situarse en un área geográfica en particular a partir de la existencia de externalidades positivas derivadas de un proceso de concentración (Santos y Teixeira, 2007).

En este sentido, el inicio de la cadena del aloe en Falcón obedeció a condiciones comparativas de su entorno: un clima y condiciones agronómicas favorables al cultivo, potenciadas por la política oficial del momento dirigida a promover la siembra de esta planta con el objetivo de extraer su resina y exportarla a Europa, donde había una gran demanda. Sin embargo, esta actividad no se realizó con un sentido de coherencia y sistematicidad, por el contrario, respondió al vaivén económicocomercial de la Colonia y sus empresas, lo que bajo el amparo de los recursos tecnológicos del momento hizo de ésta una actividad segregada, muy localizada y manejada por pocos actores, siempre bajo el dominio y la dirección de un agente foráneo con control del mercado y de las conexiones de transporte y despacho de la mercancía.

Esta tendencia prevaleció hasta mediados de la década de 1990, cuando la aparición en el mercado de una amplia gama de bienes a base de aloe hizo que su cultivo se incrementara de manera significativa: en menos de cuatro años casi se duplicó la superficie sembrada y surgieron un sinnúmero de propuestas de industrialización del cultivo; sólo llegó a materializarse la que tenía acceso al mercado y tecnología gracias a sus vínculos con capital y agentes foráneos.

En el caso del aloe, las externalidades que deberían generarse por la concentración geográfica de empresas no se producen porque no hay suficiente masa crítica de agentes (empresas) dedicados al procesamiento, que cuenten con la capacidad suficiente para impulsar una efectiva dinámica de división del trabajo, ni proveedores especializados o iniciativas conjuntas de innovación bajo un esquema de cooperación-competencia. Estas condiciones sólo se han dado, en cierto grado, en el ámbito del procesamiento primario, donde existen trabajadores semiespecializados en las labores de cosecha o 
cocción de la pasta (actividad en vías de extinción, debido al establecimiento de las plantas procesadoras) o en los centros de I\&D, con el desarrollo de prototipos mejorados para la recolección de acíbar. En ambos casos, son iniciativas orientadas a disminuir la contaminación del producto y aumentar la eficiencia del proceso.

En la cadena del aloe, la atmósfera industrial es insuficiente para generar las economías externas necesarias para fortalecer el ambiente social y de negocio del cual emerjan los beneficios de proximidad entre empresas. Tal vez una de las causas de esta situación sea el modelo de mercado derivado desde el inicio de la cadena: sólo se ha concentrado en la extracción de materia prima para su exportación, un modelo típico de producción en América Latina, tanto para productos naturales como minerales, fortaleciendo fuertes lazos cliente-proveedor, los cuales se consolidaron a lo largo del tiempo y no permitieron ni estimularon el desarrollo de un sector industrial fuerte y estructurado.

Tomando en cuenta el reciente proceso de reconversión de la empresa procesadora existente en el estado y la entrada de una nueva planta, ambos casos con un significativo y distintivo sello de capital extranjero, se asegura casi por completo el acceso a mercados y a tecnologías de punta. Estas empresas aprovechan los relativos bajos costos de transacción del transporte y la materia prima (teoría de localización) derivados de economías internas de escala situadas cerca de los centros de cultivo del aloe, favoreciendo sus retornos crecientes a escala (internos y externos), aprovechando al máximo un mercado de comportamiento marcadamente de competencia imperfecta, y potencian para sí las ventajas de localización derivadas de factores geográficos e históricos.

\section{b. Redes}

A diferencia de un conglomerado, concebido como un punto, las redes consisten en nodos y eslabones. Son complejas interconexiones de agentes. De esta manera las redes se orientan hacia la generación de proximidades: tecnológicas, de trabajo, infraestructura en común, así como a la transmisión de conocimiento y aprendizaje colectivo. La construcción de redes entre agentes de un conglomerado deriva en una estructura articulada enfocada a proveer la competitividad del territorio.

En este caso la competitividad depende sustancialmente de la capacidad del territorio de crear, acumular y utilizar conocimiento más rápidamente que sus competidores. Esta creación de conocimiento representa el nuevo factor de ventaja competitiva en la nueva economía contemporánea globalizada (Giaccaria, 1999). Debe tenerse en cuenta 
que incluso hay otros estados dentro de Venezuela (por no mencionar otros países), que compiten por inversiones en el sector del aloe.

En la cadena del aloe no existe una red formal de actores. Las conexiones se circunscriben a contactos informales entre agentes, muy puntuales en la búsqueda de solventar un aspecto de interés particular. Si bien el establecimiento de una red pudiera considerarse como el inicio, un sustituto de la fase primera del conglomerado dados los beneficios a futuro derivados de la interconexión de agentes, en términos de difusión de conocimiento o incremento de productividad (Johansson y Quigley, 2004), esta modalidad de interconexión es aprovechada por las agroindustrias privadas del estado, quienes (obvia y justificadamente) han fortalecido sus contactos aguas arriba y aguas debajo de su proceso productivo, sin que los beneficios derivados de esta actuación permeen por igual a todos los agentes de la cadena.

Es importante diferenciar redes públicas de redes privadas. Es clara la ventaja de un conglomerado a partir de su carácter público: todos tienen acceso a los mismos recursos y sus ganancias son proporcionales a la capacidad de aprovechar e internalizar esos recursos. En contraste, en una red privada sólo sus integrantes obtienen provecho. En suma, una aglomeración, a diferencia de una red privada, no excluye a ningún agente de recibir sus beneficios (Johansson y Quigley, 2004).

Ésta ha sido la situación en la cadena del aloe: el desarrollo, crecimiento y explotación primaria fueron manejados como una red privada. Por tanto, no se favoreció la construcción de un capital social e institucional capaz de promover un conglomerado alrededor de esta actividad productiva. Las iniciativas en torno al aloe se circunscribieron a la extracción de commodities para su exportación. No era beneficiosa su transformación in situ, por tanto no se promovió el establecimiento de redes públicas entre agentes, salvo las necesarias para la recolección del producto en campo y su transporte al mercado de destino.

Bajo esta situación, la red perdió su naturaleza principal para favorecer y promover un conglomerado: creación y difusión de conocimiento que derivara en el desarrollo de destrezas y competencias especializadas entre actores de la cadena y la promoción de la innovación y la mejora constantes del proceso productivo. Así, el conocimiento fue codificado paulatinamente: el resto de los actores quedaron excluidos, lo cual limitó el nacimiento del conglomerado.

Al comparar esta situación con otros conglomerados (por ejemplo, los distritos italianos), se aprecia claramente cómo el espíritu comunitario del conglomerado favoreció significativamente la creación de redes entre actores (o viceversa). En este caso una razón histórica, un saber-hacer local, fue districtualizado (Becattini, 2002) 
hasta evolucionar y configurar un ambiente promotor del conglomerado. Se fomentó un marco institucional favorable y se potenciaron valores y creencias que consolidaron las actividades emprendidas. Si bien hoy en día la cadena del aloe tiene una raíz histórica (casi 400 años de explotación), sin lugar a dudas es una actividad impuesta, foránea, implantada por una motivación particular; nunca hubo una preocupación por consolidar una estructura de producción articulada.

No se propició la formación de capital social a partir de los esfuerzos conjuntos entre actores; por el contrario, se fomentó la exclusión (en algunos casos explícitamente). No se generaron externalidades favorables asociadas a la explotación del aloe. En términos sencillos, no se desarrolló la confianza y reciprocidad entre todos los actores para favorecer al conglomerado (a excepción de la producción primaria donde los productores exhiben un clima más armónico). Se soslayó la creación de un clima de confianza mutua, que estuviera sustentado en el sentido de pertenencia e identidad en torno de una meta en común.

\section{c. Innovación}

El proceso innovador (conglomerados típicos) tiene su origen en un mecanismo de aprendizaje colectivo y de acumulación del conocimiento presente en el ambiente, del cual se deriva una circulación del saber-hacer entre empresas a partir del movimiento de mano de obra interempresas, ampliando así la capacidad local de innovación. De esta manera, el proceso innovador, generalmente materializado dentro de la empresa, deriva en una acción colectiva sobre la base de conocimiento común, acumulado más en las personas y menos en las empresas (Becattini 2002; Rabellotti, 1995).

En la cadena del aloe, el proceso descrito tiene lugar (con sus ajustes y limitaciones) sólo en el ámbito de producción primaria. El saber-hacer sobre las mejores técnicas de siembra y cosecha así como la creación artesanal de la pasta ha pasado de generación en generación, y sólo recientemente se han incorporado esquemas mejorados de cultivo, manejo de plagas y enfermedades y técnicas de cosecha creadas en los centros de I\&D del estado, desde donde se han promovido programas de modernización de la producción primaria del cultivo. Sin embargo, en el ámbito del procesamiento industrial y obtención de bienes intermedios diferenciados tales como acíbar concentrado o aloína, el conocimiento no ha fluido en la misma forma.

Esta fase de agroindustrialización en la cadena del aloe se caracteriza por su alto nivel tecnológico, de innovación. El procesamiento se realiza mediante equipos sofisticados: liofilizadores, concentradores de acíbar o evaporadores de resina, a los cuales no se tiene fácil acceso en el mercado y, en la mayoría de los casos, corresponden a 
construcciones puntuales de acuerdo con especificaciones del cliente y, ciertamente, costosas. De manera que no todos los agentes de la cadena pueden acceder a tales equipos, ya sea por su costo o por las habilidades y competencias requeridas para su manipulación. En la cadena del aloe en Falcón sólo se promovió la cocción del acíbar para obtener la pasta, como una alternativa al traslado de este líquido, ya que si no se manipula adecuadamente, el acíbar se fermenta y pierde sus tan apreciadas propiedades naturales. Por ello es que el acíbar se deshidrataba para obtener la pasta, luego se exportaba a Europa, donde el proceso se revertía y se separaban los compuestos químicos demandados del aloe: resinas y aloínas, entre otros.

Con el paso del tiempo, se han descubierto un sinnúmero de aplicaciones de distintos compuestos del aloe; simultáneamente, el proceso de innovación de este cultivo se ha vuelto más hermético, y en la actualidad está dominado por transnacionales de las industrias médica y cosmética. Tales agentes invierten grandes sumas de dinero en I\&D para crear nuevos productos, descubrir otras aplicaciones o en la mejora de los procesos, todo ello en centros foráneos desconectados de las zonas productoras de Falcón. La innovación se convierte en un conocimiento codificado, un bien privado manejado por una red privada de agentes.

En este punto se produce una ruptura de la cadena (en tanto su denominación como cadena incompleta), diferenciando claramente la producción primaria y el procesamiento intermedio (enclave de producción) y el acceso a tecnología de punta, obtención de bienes finales y conexión con el mercado (proceso innovativo). Adicionalmente, los esfuerzos de los centros de I\&D locales se han centrado en mejorar los procesos primarios de producción: siembra y cosecha, descuidando significativamente el procesamiento industrial de esa producción primaria.

Por tanto se ha instaurado un círculo vicioso que atenta contra un exitoso proceso de innovación: las condiciones primarias del aloe no favorecieron o estimularon la innovación, en consecuencia, no surgieron los mecanismos para llevar a cabo acciones de este tipo, derivando en un atrofiamiento de tal iniciativa. Hoy en día, visto el atractivo mercado del aloe, se intenta participar en el mismo, pero la realidad es contundente: resulta muy costoso, es una fase altamente diferenciada (élite, en muchos casos) y se carece de una importante infraestructura (humana y técnica) para iniciar tal tarea. En definitiva, no existe una masa crítica lo suficientemente significativa para soportar un proceso colectivo de innovación. El espacio fue copado por agentes foráneos y éstos consolidaron sus posiciones y relaciones entre actores.

En tal sentido, los mecanismos de rendimientos crecientes en la cadena del aloe están asociados a economías modernas y, en particular, a industrias basadas en el 
conocimiento, respondiendo al diseño específico de una estructura organizacional de la empresa en el conglomerado, por lo cual el mecanismo endógeno de construcción de capacidades de innovación en un esquema industrial de este tipo sigue un modelo de innovación no lineal basado en un continuum incremental de la innovación (Malmberg y Maskell, 2001), que en el caso del aloe fue truncado, nunca estimulado o promovido, al menos en el ámbito del procesamiento y obtención de bienes finales.

Ciertamente no es fácil iniciar un proceso de innovación colectiva (tal como sucede en un conglomerado típico), debido a dos factores fundamentales: el significativo nivel de especificidad del proceso en sí y los altos costos asociados al mismo; sin embargo, tampoco es imposible. La existencia de centros de I\&D y la pronta puesta en marcha de siete plantas procesadoras sin lugar a dudas configurará el punto de partida de un proceso de innovación en la cadena del aloe.

Así, al concebirse la innovación como una función compleja de un amplio rango de condiciones e interacciones entre diferentes actores, ésta toma lugar en múltiples niveles y actividades donde el nexo surgido del conglomerado favorece la innovación en una industria (Andersson, Schwaag, Sorvik y Wise, 2004). En este sentido, la introducción y difusión de un proceso productivo ampliado, el rediseño de mecanismos o el ajuste a las demandas del consumidor iniciarían mecanismos en la cadena del aloe, cuyo elemento clave sería la conexión del proceso innovador. La inversión en tecnología (innovación) en un conglomerado genera efectos sinérgicos al estimular nuevos procesos de innovación, que a su vez propician nueva innovación y, en consecuencia, nuevas inversiones, transformando el círculo vicioso en uno virtuoso.

En tal sentido, desde el punto de vista innovativo, para que la cadena del aloe en Falcón no perezca como un clásico proveedor de materia prima indiferenciada, tiene como única opción de supervivencia el escalamiento a un nivel superior agroindustrial, definitivamente debe incrementar el valor agregado de los bienes indiferenciados producidos hoy en día, y la vía lógica y natural es la evolución desde los procesos de innovación hacia la adquisición de destrezas competitivas en el conglomerado.

\section{d. Entorno institucional}

Las instituciones son reglas de juego para el funcionamiento eficaz, eficiente y competitivo de una sociedad. Son lineamientos rectores definitorios de las interrelaciones entre los seres humanos con influencia directa en el funcionamiento y desempeño del comportamiento económico de un país y, de alguna manera, ese comportamiento es el reflejo de sus interrelaciones. Son reglas del juego formales (normas o leyes) o 
informales (conductas o hábitos), y pueden ser creadas en un momento determinado o evolucionar en el tiempo desde un punto informal e institucionalizarse como norma formal (North, 2001).

En este sentido, un consolidado entorno institucional soportado en instituciones públicas y privadas de apoyo y promoción a los agentes económicos del conglomerado es decisivo para su éxito. Éste puede ser el factor clave para entender el actual comportamiento de la cadena del aloe hoy en día: siempre fue concebida como una actividad marginal, de poca importancia y restringida a unos pocos agentes económicos, que siempre han sido favorecidos. Hace sólo 20 años, a raíz del boom del aloe en el ámbito mundial, las instituciones oficiales giraron su mirada hacia esta planta, sin embargo, se trató de una visión política, no enfocada a promover su desarrollo, particularmente en los últimos 10 años. Con la promesa de modernizar la producción del aloe, se emprendieron acciones, fundamentalmente hacia la producción primaria y se utilizaron estas acciones como mecanismo de propaganda política, afectando severamente la evolución y crecimiento de la cadena.

En este contexto, debe destacarse la excelente iniciativa de creación del Grupo de Trabajo Permanente de la Zábila (GTPZ) como ente técnico de convergencia de los diferentes actores asociados al aloe y como mecanismo de planificación y coordinación de acciones a emprender en la cadena. Sin embargo, al aprobarse los montos destinados a las siete plantas procesadoras (US \$9.5 millones), la coordinación del grupo fue cercenada y el grupo extinto y olvidado. Así, una iniciativa (ciertamente oficial) surgida de un grupo importante y representativo de agentes, que en su escaso tiempo concibió y diseñó técnicamente la industrialización del aloe en Falcón y redactó un plan maestro con visión de futuro de la cadena, fue dejada de lado y otro organismo asumió el control de estas actividades bajo un matiz eminentemente político. De esta manera se desperdició una oportunidad invaluable para iniciar el desarrollo del conglomerado aloe en Falcón.

Tal como se aprecia, la participación institucional ha sido en cierta forma contraproducente y contraria a la lógica y eficiente búsqueda del desarrollo y crecimiento del conglomerado del aloe. Por otro lado, han sido los productores primarios (sector mayoritario y socialmente vulnerable) quienes han demandado mejoras sustanciales al proceso productivo y han tenido una participación preponderante en la dinámica de la cadena. Las asociaciones locales de productores son quienes principalmente han materializado las iniciativas en la cadena del aloe, que luego fueron recogidas por las organizaciones oficiales. Han sido las asociaciones los entes emprendedores líderes de la evolución de la cadena. 
Mención especial en la dinámica institucional del aloe merecen los esfuerzos promotores para lograr la declaratoria, por parte del ejecutivo regional, del aloe como rubro estratégico para el desarrollo del estado, brindando un marco institucional para el desarrollo y crecimiento de la actividad. Este instrumento jurídico ha sido subutilizado, desaprovechando sus beneficios y un marco ideal para promover iniciativas de emprendimiento en la cadena.

Como se evidencia, ha crecido progresivamente la conciencia respecto de la importancia de introducir mejoras en la explotación del aloe y del beneficio que ello reporta a toda la cadena. Estos comportamientos pueden asociarse a limitaciones informales o reglas no formales, no escritas en ningún lado, pero que con el paso del tiempo se han convertido en preceptos de obligatorio cumplimiento para todos los actores involucrados (North, 2001: 60). Así, las relaciones entre los agentes de la cadena se rigen por instituciones informales. En este sentido, la manera de producir el aloe se ha transformado en un comportamiento de individuos, materializados en normas informales de desempeño promotoras de toda una cultura del aloe en el estado, sobre la cual se ha soportado el crecimiento y desarrollo de la cadena. La participación de los organismos, organizaciones e instituciones progresivamente se ha amoldado y ha respondido a esta cultura, aunque todavía no puede calificarse de formal.

\section{De la cadena al conglomerado: supervivencia del aloe en Falcón}

Tal como se precisó al inicio del artículo, el objetivo no es la aplicación de una metodología de conglomerado (más aún cuando ésta no existe) a la cadena del aloe; por el contrario, la motivación principal ha sido poner en evidencia el desempeño del aloe en Falcón, aquellos elementos clave referidos por las investigaciones, presentes (en mayor o menor grado) en la cadena: cuáles estaban presentes, en qué cantidad y cuán fuertes son, a fin de configurar una dinámica que permita evolucionar de una cadena de valor incompleta hacia un eficiente y exitoso conglomerado.

Así, la principal aportación del artículo se centra en reconocer los elementos fundamentales para promover la evolución de la cadena del aloe hacia un conglomerado industrial, de ahí su utilidad como insumo guía para la formulación y promoción de políticas y articulación de iniciativas orientadas a este propósito.

En este sentido, en la cadena del aloe se manifestaron fuertes elementos estratégicos con incidencia determinante para la construcción del conglomerado, junto a otros igualmente fuertes que actúan en sentido contrario.

El primero de ellos es la ventaja competitiva del estado para conformar un conglomerado (condiciones agroclimáticas). Ciertamente ésta es una ventaja temporal 
y fácilmente superable, pero ha representado un elemento clave inicial de muchos conglomerados en la esfera mundial (Rabellotti, 1995). La estrategia debe centrarse en soportar la evolución competitiva del conglomerado sobre la base del aprovechamiento estratégico de esta condición.

Por otro lado, la cadena del aloe necesariamente debe dar el salto tecnológico: pasar de una simple exportación primaria de commodities a la elaboración de bienes finales de mayor valor agregado. El proceso de innovación es indispensable para lograrlo, por ello se requieren fuertes conexiones entre agentes que permitan articular una estrategia de desarrollo enfocada en un segmento específico del mercado.

Asimismo, las iniciativas empresariales relacionadas con el aloe deben concentrarse en desarrollar su aspecto comercial, donde la cadena es especialmente débil, y no sólo ocuparse del área productiva.

La cultura del aloe desarrollada entre los productores y la participación activa que han demostrado en los últimos años debe potenciarse hasta lograr articular una estrategia de coordinación, governance, sobre la cual se soporte el crecimiento del conglomerado. En este sentido, la fortaleza y calidad de las conexiones entre agentes serán determinantes para el logro de este objetivo: el espíritu comunitario será vital en el marco de una eficiencia colectiva (Schmitz, 1999). Los agentes del aloe deben aprender a cooperar para competir en un excelente y atractivo mercado.

En este contexto, la existencia de un conglomerado se sustenta en primer lugar en los beneficios derivados del intercambio de conocimiento entre agentes localizados territorialmente, lo cual no ha funcionado en la cadena del aloe; aquí radica su principal limitante: no hay un espíritu comunitario que fortalezca la confianza y reciprocidad mutua de relaciones a lo largo de todos los agentes de la cadena, incluyendo (obviamente) el procesamiento agroindustrial y la producción primaria.

En términos concretos, es claro que Falcón posee un patrón productivo de aloe sustentado en un patrimonio natural de recursos sobre los cuales se ha articulado un esquema de producción primaria, y ha descuidado los procesos de manufactura y procesamiento de la materia prima derivada de esa fase de cultivo y cosecha del aloe. Tal situación ha limitado históricamente la aparición de mecanismos de redes dirigidos a crear un ambiente propicio para configurar programas de innovación y desarrollo tecnológico basados en el saber-hacer local, en el transcurso de la evolución histórica del cultivo en el estado.

En la cadena del aloe en el estado de Falcón no hay un argumento vertebrador que aglutine e identifique a los agentes participantes; esta carencia impide estimular la construcción de un andamiaje institucional de apoyo a las iniciativas emprendedoras 
dentro de la cadena, que dé origen al entramado de relaciones para generar los beneficios de un esquema de organización industrial tipo conglomerado.

Finalmente, es importante destacar que el estudio de los conglomerados debe enfocarse como forma de organizar la producción y las actividades productivas. Es una unidad de análisis, por tanto, no existen formas idénticas de organización. Cada conglomerado es único, al igual que cada perspectiva de estudio. Por otro lado, aunque muchos estudios de conglomerados sean más historias que estadísticas (Head, Ries y Swenson, 1995), debe tenerse en cuenta que las complejas relaciones humanas (centro de los conglomerados) son historias construidas día a día por los agentes económicos de un conglomerado, en muchos de los cuales no se producen estadísticas que cuantificar, sino historias que documentar y valorar.

\section{Bibliografía}

Andersson, Thomas, Silvia Schwaag, Jens Sörvik y Emily Wise, Cluster Policies Whitebook, Malmö, International Organisation for Knowledge Economy and Enterprise Development, 2004.

Becattini, Giacomo, "Industrial sectors and industrial districts: tools for industrial analysis”, European Planning Studies, vol. 10, núm. 4, 2002, pp. 483-493.

Boscherini, Fabio y Lucio Poma, Territorio, conocimiento y competitividad de las empresas. El rol de las instituciones en el espacio global, Buenos Aires, Miño y Dávila, 2000.

Giaccaria, Paolo, "Learning and local competitiveness: the case of Turin", Geojournal, vol. 49, núm. 4, diciembre de 1999, pp. 401-410.

González, Carlos, Antecedentes históricos del cultivo de la zábila en Falcón, Santa Ana de Coro, Fundacite Falcón, 1999.

Head, Keith, Jhon Ries y Deborah Swenson, "Agglomeration benefits and location choice: evidence from Japanese manufacturing investments in the United States", Journal of International Economics, vol. 38, núms. 3-4, mayo de 1995, pp. 223-247.

Johansson, Börge y Jhon Quigley, "Agglomeration and networks in spatial economics", Paper in Regional Science, vol. 83, núm. 1, enero de 2004, pp. 165-176.

Krugman, Paul, "Increasing returns, monopolistic competition and international trade", Journal of International Economics, vol. 9, núm. 4, noviembre de 1979, pp. 469-479.

Vol. 41, núm. 160, enero-marzo / 2010
Malmberg, Anders y Peter Maskell, "The elusive concept of localization economies: towards a knowledge based theory of spatial clustering", en Industrial Clusters' Revisited: Innovative Places or Uncharted Spaces?, Nueva York, Association of American Geographers Annual Conference, 2001.

Marshall, Alfred, Principles of Economics, $8^{\mathrm{a}}$ ed., Londres, Macmillan, 1920.

North, Douglas, Instituciones, cambio y desempeño económico, México, Fondo de Cultura Económica, 2001.

Piña, Henri, "El circuito zábila (Aloe vera L.) en el estado Falcón, Venezuela", en Adolfo Álvarez, François Boucher, Fernando Cervantes, Angélica Espinoza, José Muchnik y Denis Requier, Agroindustria rural y territorio, tomo I: Los desafíos de los sistemas agroalimentarios localizados, México, Universidad Autónoma del Estado de México, 2006, pp. 229-254

"Perfil preliminar del mercado de la zábila (Aloe barbadensis Mill.) en el estado Falcón, Venezuela", Bioagro, vol. 17, núm. 2, 2005, pp. 85-92.

"El conglomerado zábila (Aloe vera L.) en el estado Falcón, Venezuela", Cuadernos de desarrollo rural, núm. 53, 2004, pp. 37-57.

"Relaciones interindustriales en el circuito zábila en el estado Falcón", Agrociencia, 2008 (en arbitraje). 
Piña, Henri, Zunilde Lugo, Rhode Azócar y Carlos Romero, "Tipología de la producción primaria de zábila (Aloe vera $L$.) en el estado Falcón, Venezuela", Bioagro, vol. 17, núm. 1, 2005, pp. 25-34.

Porter, Michael, "Clusters and the new economics of competition", Harvard Business Review, nov.-dic. de 1998, pp. 77-90.

Rabellotti, Roberta, "Is there an "industrial district model'? Footwear districts in Italy and Mexico compared", World Development, vol. 23, núm. 1, 1995, pp. 29-41.
Santos, Sara y Aurora Teixeira, A New Look into the Evolution of Clusters Literature. A Bibliometric exercise, Universidad de Porto, 2007.

Schmitz, Hubert, "Global competition and local cooperation: success and failure in the Sinos Valley, Brazil", World Development, vol. 27, núm. 9, 1999, pp. 1627-1650.

Sotolongo, Pedro, "Complejidad, no linealidad y redes distribuidas", Complexus. Revista de complejidad, ciencia y estética, vol. 3, núm. 1, marzo de 2007.

\section{Desarrrollo}

